\title{
Impedance determination of terminal power amplifiers for optimal antenna matching using load-pull method
}

\author{
Prasadh Ramachandran ${ }^{(1,2)}$, Olof Bengtsson ${ }^{(2)}$, and Claes Beckman ${ }^{(2)}$ \\ (1) Pulse Finland Oy, Takatie 6, 90440 Kempele, Finland \\ PrasadhRamachandran@pulseeng.com \\ (2) University of Gavle, Centre for RF Measurement Technology \\ Gävle, SE-801 67, Sweden \\ bob@hig.se \\ cbn@hig.se
}

\begin{abstract}
In this paper we present a single tuner based load-pull method for characterization of optimal antenna load impedance in mobile terminals. In the proposed load-pull set-up the mobile terminal itself is used as a source. The presented method can provide valuable data for antenna design since it determines the optimal impedance at the antenna connector/pad taken into account the full output circuitry of the mobile terminal. It reveals the modification of the power amplifier, PA, output impedance caused by the presence of SAW filter, antenna switch, and transmission line possibly present between the PA and the antenna. With the presented method it is not only possible to characterize the optimum antenna impedance to be presented to the terminal' PA in different bands and channels but also to reveal the impedance miss-match sensitivity of the PA for each channel.
\end{abstract}

\section{INTRODUCTION}

Today's mobile communication networks require highly efficient and reliable mobile terminal performance since its radio performance may reduce the coverage and/or the capacity of the whole network, [1]. In a mobile phone, the single block which consumes most of the power is the Power Amplifier, (PA). For low current consumption it is important to keep the transmitted power at lowest possible level with sufficient connection. This is possible when the antenna, which is the load for the PA, is matched perfectly to the PA's output impedance. Any mismatch between the PA and antenna will reduce the transmitted power and the base station will force the terminal to increase output power in order to maintain connection. The increased output power cause higher current consumption with reduced talk-time as a consequence. The individual system blocks on the terminal output, like PA, filter and antenna, should all be matched to 50-ohm. In reality, none of these blocks are ideal 50-ohm loads. In addition, the SAW filter, switch and transmission lines connecting the modules, contribute to an overall frequency-band dependant delay. This delay can be observed at the antenna interface as a rotation (phase shift) in the Smith chart producing a change of the optimum impedance for which to match the antenna.

Assuming the individual system blocks on the output to have a constant delay they can be modeled as a transmission line and the output impedance of the PA, as seen by the antenna, is given by equation 1 [2]:

$$
Z_{i n}=Z_{O} \frac{Z_{S}+j Z_{o} \tan \beta l}{Z_{O}+j Z_{S} \tan \beta l}
$$

where $\beta=2 \pi / \lambda ., Z_{0}=50 \mathrm{ohm}, Z_{s}=$ Output impedance of the PA, and $l=$ transmission line length

From equation (1) it can be seen that the impedance is a function of $l$ (the delay), the frequency and the PA's output impedance. The delay is band-dependant due to the individual system blocks and different signal paths on the terminal output stages. The delay will also be different for every mobile terminal platform since the location of the different components change based on the features included.

Further, the output impedance of the PA, is a function of frequency and operating power level. These factors cause the impedance at the antenna connector/pad to vary significantly. The change in impedance in each band due to the output circuitry phase shift may cause significant deterioration in performance if not properly considered in the antenna design. Insufficient knowledge of these effects creates a need to make several trials in the antenna optimization process to meet the total radiated power (TRP) requirements for all the bands. This translates into long development times and high development cost. The presented method provides a fast solution to attain crucial data for antenna optimization at individual bands. 


\section{LOAD-PULL MEASUREMENT SYSTEM}

Load-pull is a classical approach to measure device performance as a function of impedance under non-50 ohm conditions. Impedances different than 50 -ohms are generated with excellent repeatability and accuracy using precalibrated stepper engine controlled mechanical tuners where movable slugs are used to alter the impedance to a predefined value. Load-pull systems are traditionally used to characterize two-port system with regards to e.g. gain, PAE or noise [3]. For such measurements the tuners generating the altered impedance are used together with a signal generator providing the stimuli-signal for the measurement.

In this work a simplified one tuner load-pull measurement setup is proposed where measurements are made on a mobile terminal PA using the terminal itself as generator for the system. The measurement system can be depicted as the following block diagram:

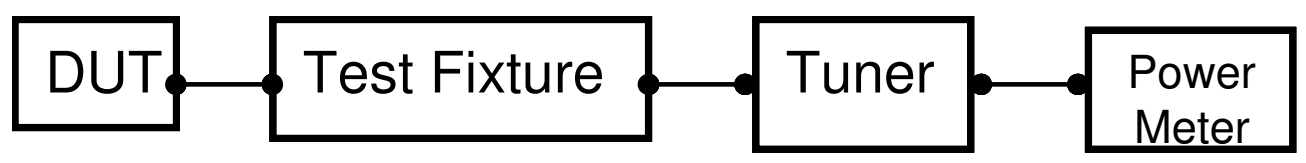

Fig. 1 Schematic illustration of the single tuner Load pull set up

In the block diagram, the $D U T$ represents the mobile terminal. The tuner that generates the required mismatch is a MT982 from Maury Microwave optimized for high reflection coefficients at telecom frequencies. The test fixture is the probe/adapter used to reach the antenna test connector from the tuner. A solid mechanical construction is used to fix it to the terminal in a repeatable manner. A power meter (here a Gigatronic 8541C with Power Sensor 80401A) is used to measure the power level of the GSM-TDMA signal from the PA towards the load. The standard Maury-ATS software is used to control the impedance position of the tuner and to make a calibrated measurement of the output power from the DUT.

\section{CALIBRATION}

The overall system calibration consists of three major parts, tuner calibration, test-fixture de-embedding and system power calibration.

- Tuner Calibration: The tuner is calibrated at the selected frequencies in the low, mid, and high channels of the respective GSM bands for a predefined number of impedance positions using a highly accurate vector network analyzer, VNA. For increased accuracy the VNA is calibrated using a through-reflect-line, TRL, method.

- Test-fixture de-embedding: The Maury-ATS fixture characterization software is used here to characterize the test fixture (adapter) between the antenna connector and the tuner. An S21 measurement is made of two joint adapters, SMA- Antenna (male) and Antenna (female)-SMA. From that measurement the overall group delay is found. With the assumption that the two adapters are "equal" i.e. delay and loss is equal in both the adapters, the adaptor response is found which is then used to shift the reference plane to the DUT interface also taking the adapter losses into consideration.

- System Power Calibration: The power sensor mismatch is taken into account by measuring S11 at the power sensor interface. This information together with possible loss from attenuators added to the power sensor is entered in Maury-ATS and then compensated automatically by the software during load-pull measurement when power level is measured. For proper GSM-TDMA characterization the power meter was configured for burst-mode time-slot power measurements.

\section{MEASUREMENTS AND RESULTS}

Measurement were made on a mobile terminal with a special software to select the transmit channel at the maximum power level. The optimum load impedance for maximum output power for some channels in the three GSM bands are shown in Table 1. The possibility of making an optimum match shows that the terminal is capable of delivering more power than what is specified for the different bands. The GSM1900 band for e.g. is specified at 30dBm but the terminal is capable of producing well above $31 \mathrm{dBm}$ which gives a margin for antenna miss-match losses. Figure 1 shows the optimum impedance matching point rotation with frequency for the GSM900 and GSM1800 band. This rotation is what the antenna needs to track in order to remain properly matched over the frequencies. 


\begin{tabular}{|c|c|c|c|c|}
\hline & Frequency $[\mathrm{MHz}]$ & Pout of PA $[\mathrm{dBm}]$ & $\Gamma$ & $\mathrm{Z}[\Omega]$ \\
\hline \multirow{2}{*}{$\sum_{\substack{\Delta \\
0}}$} & 880.2 & 33.3 & $0.25<12^{\circ}$ & $81.4+8.8 \mathrm{i}$ \\
\hline & 897.6 & 33.5 & $0.24<35^{\circ}$ & $70.9+20.9 i$ \\
\hline \multirow{3}{*}{ 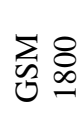 } & 1710 & 30.4 & $0.34<-94^{\circ}$ & $38.2-29.1 \mathrm{i}$ \\
\hline & 1747.8 & 30.5 & $0.25<-31^{\circ}$ & $74.2-20.6 \mathrm{i}$ \\
\hline & 1784.8 & 30.4 & $0.35<24^{\circ}$ & $87.6+25.6 i$ \\
\hline \multirow{3}{*}{ 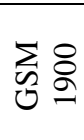 } & 1850.2 & 31.6 & $0.35<83^{\circ}$ & $42.5+33.1 \mathrm{i}$ \\
\hline & 1880 & 31.4 & $0.41<146^{\circ}$ & $22.4+12.4 \mathrm{i}$ \\
\hline & 1990.8 & 31.2 & $0.48<166^{\circ}$ & $18.0+5.3 \mathrm{i}$ \\
\hline
\end{tabular}

Table 1. Results of Load-pull measurement

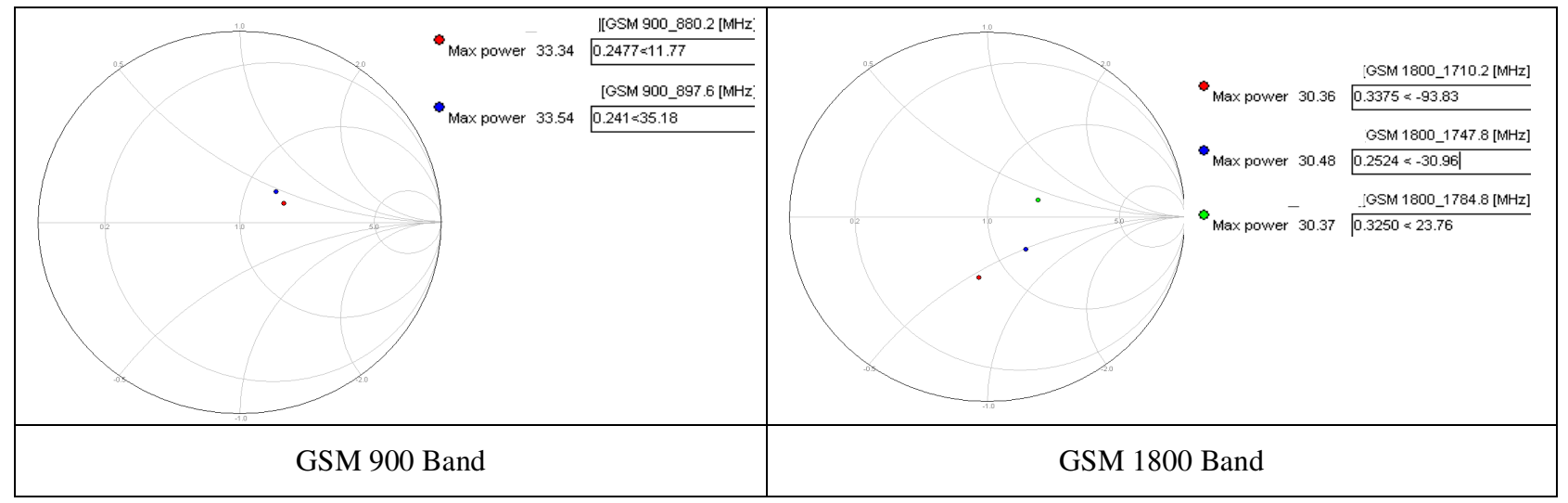

Figure 1. Smith chart representation of the load-pull measurement results

A more detailed study of the impedance charts with constant output power contours, Figure 3, reveals the impedance matching sensitivity for the different channels and bands.

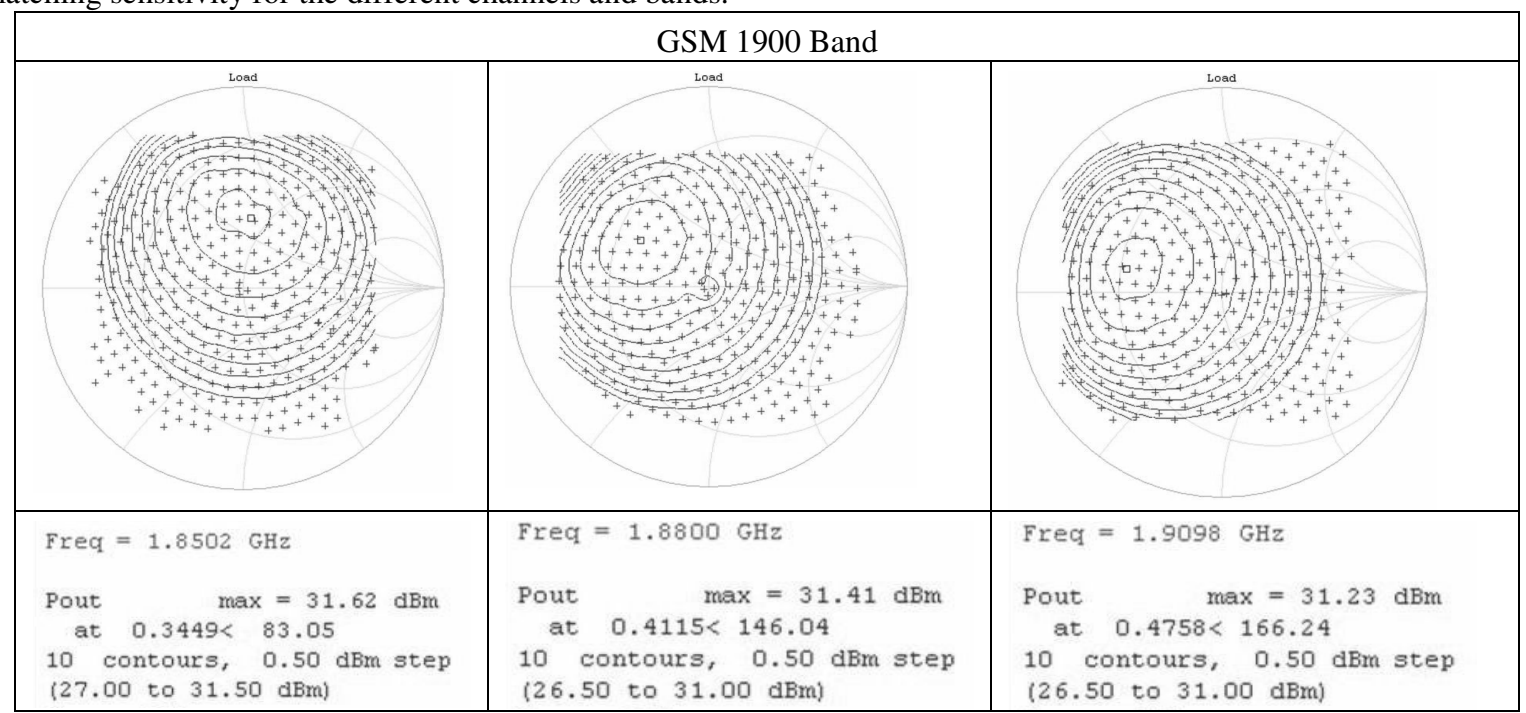

Figure 2. Smith chart with power contours of the load-pull measurement results

For the GSM1900 band shown, the slightly higher output power in the lower channel compared to the higher channel $31.6 \mathrm{dBm}$ compared to $31.2 \mathrm{dBm}$, gives a much larger impedance matching region (less sensitive) for a constant output power. (Compare the area of the second contour of the lower channel at $31 \mathrm{dBm}$ with the first contour of the higher channel at $31 \mathrm{dBm})$ 
The repeatability of the measurement system was tested by performing calibration and measurements by two operators. The variation in magnitude was observed to be $0.05 \mathrm{~dB}$ and the variation in phase was a maximum of $2^{\circ}$ for the lowband and maximum of $30^{\circ}$ in the high band.

\section{DISCUSSION}

The results in the Table 1, show the load impedance that the source (PA) should see at the antenna connector/pad, needs to see in order to achieve a "match" and hence deliver maximum power. This information can be directly used to reduce the losses due to the difference in the antenna impedance and the optimal impedance to be seen at the pads.

In a linear system the mismatch losses are given by Equation 2. According to the equation phase difference of $90^{\circ}$ between optimal impedance and the impedance at the pads will cause a mismatch loss of about $3.8 \mathrm{~dB}$, which is very close to the values measured using the system. If the resistive part of the impedances, $R_{a n t}=2 \times R_{\text {optimal }}$, a mismatch loss close to $6 \mathrm{~dB}$ will be observed in a linear system, but the losses denoted from the load-pull system is close to 1.5 $2 \mathrm{~dB}$. This analysis gives us information on the direction in which the antenna impedance needs to be modified to obtain a better performance thus contributing to the overall system performance.

$$
\text { MissmatchLoss }=-10 \log _{10}\left(1-\Gamma^{2}\right)
$$

The measurement method also gives us the indication of the sensitivity of the PA for impedance variation based on the frequency. This effect observed from Table 3, shows us that even within the band as the frequency increases the $0.5 \mathrm{~dB}$ loss contour reduces in dimension indicating that for higher frequency even a small variation in the load impedance can give a big variation in the power delivered.

The proposed load-pull measurement setup gives us the possibility to characterize the terminal in a very efficient way despite being slightly cumbersome owing to some difficulties faced during calibration, which are:

- The phase error was difficult to characterize owing to the fixture calibration method.

- Stability of the connection between the adapter and the phone's test connector/pad. A setup with mechanically controlled probe will be a good approach to reduce this error.

The next generation mobile systems like LTE, which support packet driven network for all applications, will put great stress on the overall performance of the handsets. A key feature of these future mobile networks is its agile nature, which calls for variable data rate, variable bandwidth and variable power level based operations based on the user's requirements [4]. This has created a lot of interest in tunable PAs, wherein techniques like load modulation [5], bias modulated PAs are studied, which affect their output impedance for different operation conditions.

On the antennas front, key elements in determining the performance are the efficiency of antenna and the PAE which points to the power delivered by the PA to the antenna (load). A significant amount of effort is being put into increasing the PAE, for which impedance variable antenna are proposed to "match" the antenna to the PA. The proposed measurement method gives us exactly the required information.

\section{CONCLUSIONS}

In this paper we have presented a novel terminal measurement method capable of determining the impedance characteristics of a terminal for different frequency bands and power levels. The method also provides a detailed insight into sensitivity at the individual bands, to the impedance variation of the antenna. This invaluable data can give much needed information for the antenna design. Even though the calibration procedure is cumbersome, our initial results are very promising indicating that the load-pull measurement technique could become an important tool for the antenna engineer in order to optimize the power added efficiency of future mobile phones.

\section{REFERENCES}

[1] 3GPP TSG-RAN Working Group 4 (Radio) meeting \#27, R4-030546, Paris, France 19th -23rd May, 2003, "UE antenna efficiency impact on UMTS system coverage/capacity"

[2] David M. Pozar, "Microwave Engineering", $3{ }^{\text {rd }}$ Edition, Wiley International 2005

[3] Maury Microwave Corporation. [Online]. Available: http://www.maurymw.com/.

[4] 3G Evolution (HSPA and LTE for Mobile Broadband, Erik Dahlman, Stefan Parkvall, Johan Sköld, Per Beming, Academic Press, 2007

[5] Fabien Lepine, Rik Jos, Hebert Zirath, "A load modulated high efficiency power amplifier", $36^{\text {th }}$ European Microwave Conference. September 2006. 